\title{
c-jun Is Dispensable for Developmental Cell Death and Axogenesis in the Retina
}

\author{
Karl-Heinz Herzog, Shu-Cheng Chen, and James I. Morgan \\ Department of Developmental Neurobiology, St. Jude Children's Research Hospital, Memphis, Tennessee 38105
}

\begin{abstract}
Although a number of studies have implicated c-Jun in neuronal death and axonal regeneration, it is unknown whether Jun function is essential for either response. One approach to resolve this issue is to analyze knock-out mice. However, c-junnull mice die at midgestation, precluding critical investigation. Therefore, a xenograft paradigm was used in which retinas from embryonic day 12.5 (E12.5) c-jun nullizygous or wild-type mice were transplanted onto the superior colliculus of newborn rats. The rats were allowed to develop, and the grafts were assayed at various times for cell death and axon growth. Histologically, grafts of both genotypes developed in identical manners and had morphological characteristics of retinas. A functional c-jun allele was not essential for axogenesis, because ganglion cells in retinal grafts from c-jun nullizygous mice developed axons
\end{abstract}

Expression of c-jun has been associated with neuronal death (Estus et al., 1994; Guégan et al., 1997) and regeneration (Jenkins and Hunt, 1991; Leah et al., 1991; Vaudano et al., 1998). However, the role of c-jun in these two apparently opposing responses is controversial.

Frequently, neurons that contribute to peripheral nerves can regrow their axons after axotomy. This regenerative response is accompanied by a rapid upregulation of c-jun expression (Jenkins and Hunt, 1991; Leah et al., 1991; Herdegen et al., 1993; Jenkins et al., 1993; DeFelipe and Hunt, 1994; Vaudano et al., 1998) that generally persists until the neuron reestablishes a contact with its target field (Jenkins and Hunt, 1991; Leah et al., 1991; Herdegen et al., 1993). In contrast, neurons that are confined to the CNS cannot normally regrow their axons and express little or no c-jun after axotomy. However, when these neurons are provided a peripheral nerve graft that permits axogenesis, they robustly express c-jun (e.g., Vaudano et al., 1998). Despite these strong circumstantial links between c-jun expression and axonal growth, their significance has been questioned (e.g., Isenmann and Bähr, 1997).

Expression of c-jun has also been observed in association with neuronal death (Dragunow et al., 1993; Estus et al., 1994; Anderson et al., 1995; Ferrer et al., 1996; Guégan et al., 1997). Additionally, overexpression of c-Jun triggers the death, whereas ap-

\footnotetext{
Received Oct. 30, 1998; revised March 10, 1999; accepted March 17, 1999.

This study was supported in part by National Institutes of Health Cancer Center Support CORE Grant P30 CA21765 and by the American Lebanese Syrian Associated Charities. K.-H.H. was partly supported by the Deutsche Forschungsgemeinschaft.

Correspondence should be addressed to Dr. James I. Morgan, Department of Developmental Neurobiology, St. Jude Children's Research Hospital, 332 North Lauderdale Street, Memphis, TN 38105.

Dr. Chen's present address: Schering-Plough Research Institute, 2015 Galloping Hill Road, Kenilworth, NJ 07003.

Copyright (C) 1999 Society for Neuroscience 0270-6474/99/194349-11\$05.00/0
}

that projected into the colliculus. Programmed cell death (PCD) was also evident in the age-appropriate regions of the retina in both wild-type and c-jun-null grafts. Furthermore, there were no discernible differences in the number or location of dying cells in the two genotypes. That c-jun was not essential for PCD was supported by two additional findings. First, a c-jun-lacZ reporter gene was expressed in many cells in developing and grafted retinas, although only a few of these cells were destined to die. Second, in E12.5 c-jun-null embryos there were normal levels of PCD in the trigeminal ganglion. Together, these data indicate that C-Jun is not essential for axon growth in the retina or for PCD in the retina and trigeminal ganglion.

Key words: c-jun; AP-1; knock-out mice; programmed cell death; axogenesis; retinal grafts plication of either neutralizing antibodies to c-Jun or dominantnegative forms of the transcription factor suppresses the death of cultured sympathetic neurons after growth factor withdrawal (Estus et al., 1994; Ham et al., 1995). Moreover, overexpression of c-Jun (Bossy-Wetzel et al., 1997) or administration of antisense oligonucleotides to c-fos and c-jun (Colotta et al., 1992) modify death in other cell types; suggesting a wider involvement in cell killing. However, programmed cell death (PCD) among dorsal root ganglion neurons in c-jun-null embryos is normal (RofflerTarlov et al., 1996). Thus, although there are strong causal links between c-Jun function and death in culture, it is unclear whether c-jun is essential for cell killing in vivo.

One way to resolve whether c-jun is essential for neuronal death or regeneration is through the analysis of c-jun-null mice. However, c-jun-null embryos die at approximately embryonic day 12.5 (E12.5) (Hilberg et al., 1993; Johnson et al., 1993), limiting the investigation of these two processes. Therefore, to extend the survival of c-jun-null neural tissue, a retinal xenograft paradigm was used (McLoon and Lund, 1980). This system was selected because (1) the timing, levels, and location of cell death in the developing retina are documented (Young, 1984; Horsburgh and Sefton, 1987); (2) c-jun expression has been associated with axonal sprouting and death in the retina after axotomy (Herdegen et al., 1993; Koistinaho et al., 1993; Hüll and Bähr, 1994a,b; Robinson, 1994, 1995, 1996; Isenmann and Bähr, 1997); and (3) ganglion cells within the xenografts generate axons, providing an opportunity to study the influence of c-Jun on axogenesis. It is shown that cell death and axon growth are indistinguishable in retinal grafts from wild-type and c-jun-null mice.

\section{MATERIALS AND METHODS}

Animals. Mice and rats were maintained in microisolator cages with continuous food and water supply. For the transplantation studies, CD rats (Charles River Laboratories, Wilmington, MA) were used as hosts. 
Mice with a heterozygous deletion in the c-jun gene (The Jackson Laboratory, Bar Harbor, ME) were bred onto a C57Bl/6J background. The heterozygotes were mated to generate c-jun nullizygous, c-jun heterozygous, and c-jun wild-type embryos for donor retinas. A transgenic mouse line (c-jun-lac Z), in which the promoter region of c-jun drove expression of bacterial $\beta$-galactosidase (S.-C. Chen and J. I. Morgan, unpublished data) was used as a surrogate means to monitor c-jun expression in retinal grafts. Another line of transgenic mice (thymosin$\beta 10$-lacZ), which harbored bacterial $\beta$-galactosidase under the control of the thymosin- $\beta 10$ promoter (Chen and Morgan, unpublished data) was used to assess path finding of grafted retinal ganglion cells. Both transgenic mouse lines were on a B6C3 background.

Genotypic analysis of embryos. For the grafting studies, c-jun heterozygous mice were mated, and embryos were removed from the mother between E11.5 and E13.5. Genotypic analysis of mouse embryos was performed on tails according to the supplier's protocol (The Jackson Laboratory). In brief, embryonic tails were digested in $250 \mu \mathrm{g} / \mathrm{ml}$ proteinase K (Boehringer Mannheim, Indianapolis, IN) for $2 \mathrm{hr}$. After denaturation, PCR was performed with primers IMR006 (GCT AGC ACT CAC GTT GGT AGG) and IMR007 (CTT CCA CCG AGA ATT CCG TGA) to detect the wild-type c-jun allele. Primers IMR008 (AGC GGT TCC TTG GAG CCC GC) and IMR009 (TAA AAC GCA CGG GTG TTG GGT), which amplify the junction of c-jun and the neomycin resistance gene, were used to identify the disrupted allele.

Grafting experiments. Mouse embryos (11.5 to 13.5 d old) were removed by cesarean section from deeply anesthetized (ketamine and xylazine) pregnant $\left(\mathrm{c}-j u n^{+-}\right)$females. After the procedure, the latter were killed by cervical dislocation, whereas embryos were decapitated. Eyes were immediately dissected from the embryos, and the adherent pigmented epithelium was removed with fine surgical forceps. As hosts, postnatal rats [postnatal day $0(\mathrm{P} 0)$ ] were deeply anesthetized by hypothermia, and a small incision was made into the skin and the skull above the left superior colliculus. The donor retina was drawn directly into a glass micropipette connected to a Hamilton syringe filled with F-10 medium. The graft was gently ejected onto the right superior colliculus after inserting the tip of the pipette into the incision. Subsequently, the wound was sealed with a suture strip. After the operation, rat pups were warmed on a heating pad and brought back to the mother. They were constantly monitored during the recovery period. A total of nine ${\mathrm{c}-j u n^{+/+}}$, three c-jun ${ }^{+/-}$, and eight $\mathrm{c}-\mathrm{jun}^{-1-}$ grafts were analyzed for general morphology, axon formation, and cell death in this study. In addition, two retinal grafts each from c-jun-lac $\mathrm{Z}$ and thymosin- $\beta 10-$ lac $\mathrm{Z}$ mice were examined by $\beta$-galactosidase histochemistry. All figures are representative examples from grafts of the indicated genotypes. All animal procedures were approved by the St. Jude Institutional Animal Care and Use Committee and conformed to all appropriate St. Jude and National Institutes of Health guidelines.

Histological analysis. After various lengths of time, animals were deeply anesthetized with ketamine and xylazine and transcardially perfused with $2 \%$ paraformaldehyde (PFA) for $\beta$-galactosidase histochemistry. Animals were post-fixed with $2 \%$ PFA in PBS for $3 \mathrm{hr}$. After sucrose impregnation (20\% sucrose in PBS) for $24 \mathrm{hr}$, tissues were frozen, and cryostat sections were thawed onto charged slides (Fisher Scientific, Pittsburgh, PA). For $\beta$-galactosidase histochemistry, slides were incubated overnight in the presence of 5-bromo-4-chloro-3-indolyl$\beta$-D-galactopyranosidase (X-gal; Boehringer Mannheim) and counterstained with neutral red. For in situ end labeling (ISEL), cryosections were post-fixed in $4 \%$ paraformaldehyde for $10 \mathrm{~min}$ followed by ethanol/ acetic acid $(2: 1)$ fixation at $-20^{\circ} \mathrm{C}$. After proteinase $\mathrm{K}$ treatment $(10$ $\mu \mathrm{g} / \mathrm{ml}$ ) for $10 \mathrm{~min}$, sections were bleached in $2 \%$ hydrogen peroxide for $5 \mathrm{~min}$ to quench endogenous peroxidase. Fragmented DNA was labeled with the Apoptag kit (Oncor, Gaithersburg, MD) according to the manufacturer's protocol. Slides were counterstained with methyl green and mounted in Permount (Fisher). For double labeling of Jun-lacZ and ISEL, slides were processed first for $\beta$-galactosidase histochemistry followed by in situ end-labeling technique.

The silver-staining technique was performed according to the method of Ungewitter (1951). Cryosections were processed through an ethanol series up to $80 \%$. Slides were then incubated for $90 \mathrm{~min}$ in Ungewitter's urea silver nitrate solution consisting of $10 \mathrm{ml}$ of $2 \%$ aqueous silver nitrate, $10 \mathrm{ml}$ of distilled water, $5 \mathrm{gm}$ of urea, and $30 \mu \mathrm{l}$ of aqueous mercuric cyanide-picric acid (1\% mercuric cyanide and $1 \%$ picric acid). After two washes in distilled water, sections were treated with reducing reagent $(10 \%$ anhydrous sodium sulfite, $2 \%$ hydroquinone, and $25 \%$ urea) and processed for coverslipping.
For quantitative analysis of cell death, pyknotic nuclei were counted by bright-field microscopy of neutral red-stained sections of c-jun ${ }^{+/+}$retinas or retinal grafts from c-jun ${ }^{+/+}$or c-jun ${ }^{-/-}$embryos. The entire graft was serially sectioned for analysis. Subsequently, five sections (spaced every 10 sections) were selected from each graft that spanned the central portion of the retina. The total number of pyknotic nuclei per section was then counted. These data are presented as the mean number of pyknotic nuclei per square millimeter \pm SEM. A total of four grafts per genotype were analyzed.

Western blot analysis. Tissue samples were extracted in $50 \mathrm{~mm}$ Tris$\mathrm{HCl}, \mathrm{pH} 7.5$, containing $250 \mathrm{~mm} \mathrm{NaCl}, 0.1 \%$ NP-40, 1 mм EDTA, 20\% glycerol, $5 \mathrm{~mm}$ DTT, and protease inhibitors (Complete; Boehringer Mannheim). Control fibroblast extracts containing nonphosphorylated and phosphorylated c-Jun were obtained from New England Biolabs (Beverly, MA). Fifty micrograms of protein were loaded onto $12 \%$ acrylamide gels. After electrophoresis and electroblotting onto nylon membranes, the latter were incubated with a rabbit polyclonal anti-c-Jun antiserum (Ab-1, 1/100; Calbiochem, La Jolla, CA). Bound immunoglobulin was detected with the ECL system (Amersham, Amherst, MA).

\section{RESULTS}

\section{Lack of a functional c-jun allele does not affect axogenesis in the E12.5 embryo}

Because c-jun-null mice die in midgestation (Hilberg et al., 1993; Johnson et al., 1993), it was not possible to test directly whether c-jun was essential for axonal regeneration in the adult. However, it is known that there are a number of biochemical responses that are common to regenerating neurons in the adult and immature neurons undergoing axogenesis during development (Gorgels et al., 1987; Van der Zee et al., 1989; Pfenninger et al., 1991). Therefore, as an approximation to regeneration, the dependence of developmental axonal growth on c-Jun was investigated in c-jun-null mice.

Both wild-type and c-jun-null E12.5 embryos were examined for axon growth using Ungewitter's silver-staining method (Ungewitter, 1951). Although axons in the E12.5 embryos did not stain as intensely as they did in either older animals or differentiated retinal grafts, they were readily detected in the CNS of both wild-type and c-jun-null mice. In coronal sections through the hindbrain near the floor plate, commissural fibers were apparent in both wild-type and c-jun-null embryos (Fig. 1a,b). Moreover, there were no obvious differences in the location or physical characteristics of axons in this and other brain regions of embryos of either genotype (Fig. $1 a, b$; data not shown). Therefore, a functional c-jun allele was not necessary for the formation and path finding of axons in the E12.5 embryo.

\section{Lack of a functional c-jun allele does not affect apoptosis in the E12.5 embryo}

The embryonic lethality of c-jun-null mice also limits their use in the analysis of PCD, because much of the growth factordependent cell death in the nervous system occurs at later stages of development (Wright et al., 1983; Young, 1984; Herrup and Busser, 1995). Nevertheless, one previous study showed that PCD among DRG neurons was normal in c-jun-null embryos at E12.5 (Roffler-Tarlov et al., 1996). Therefore, ISEL (Gavrieli et al., 1992) was used to determine whether cell death was affected in other areas of the nervous system in c-jun-null embryos.

ISEL-positive cells were detected throughout the heads of E12.5 embryos of both genotypes in approximately equal numbers and distributions. Apoptotic cells were particularly abundant in the developing inner ear, lamina terminalis, and trigeminal ganglia (Fig. 1c,d; data not shown). Apoptotic cells were also evident in the telencephalic vesicle and pallidum (data not shown). Thus, the absence of a functional c-jun allele did not 


\section{C-jun+l+}
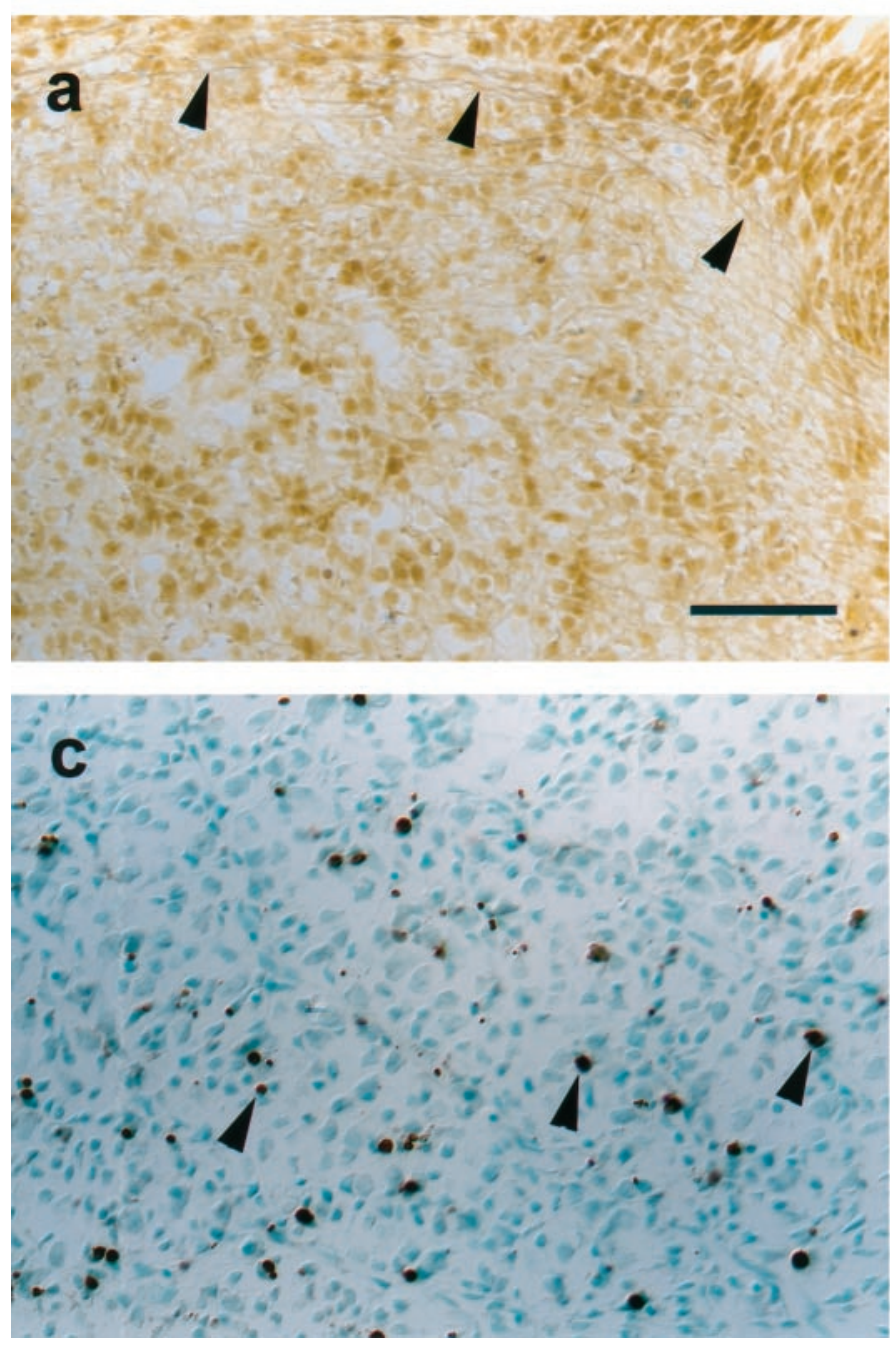

Figure 1. Axogenesis and developmental cell death in E12.5 c-jun ${ }^{+/+}$and c-jun ${ }^{-/-}$embryos. Ungewitter's staining of coronal sections through the hindbrains of c-jun ${ }^{+/+}(a)$ and c-jun ${ }^{-1-}(b)$ E12.5 embryos reveals the presence of commissural axons (arrowheads). Note that there are no obvious differences in the appearance or positions of axons in the two genotypes. ISEL staining (brown reaction product, arrowheads) revealed extensive death in the E12.5 trigeminal ganglion $(c, d)$. However, the trigeminal ganglia of wild-type $(c)$ and c-jun knock-out $(d)$ embryos did not display any obvious differences in the number or anatomical positions of dying cells. Scale bar, $50 \mu \mathrm{m}$.

appear to affect PCD, as determined by ISEL, in either the CNS (e.g., telencephalic vesicle) or PNS (e.g., trigeminal ganglion) of midgestational embryos.

The stimuli that trigger apoptosis in early neurodevelopment may not be the same as those operating at later stages, at which target-dependent selection and synaptic competition play a role (Oppenheim, 1991; Blaschke et al., 1996). Moreover, the causal experiments that linked c-Jun to neuronal death involved growth factor dependence of sympathetic neurons isolated around the time of birth (Estus et al., 1994; Ham et al., 1995). Thus, the results obtained in E12.5 c-jun-null embryos may not be relevant to the latter form of growth factor-dependent PCD. Similarly, it cannot be precluded that axogenesis is initiated in c-jun-null mice, but that axons subsequently degenerate. Therefore, a retinal xenograft paradigm was used to study the influence of c-jun on cell death and axogenesis during later development.

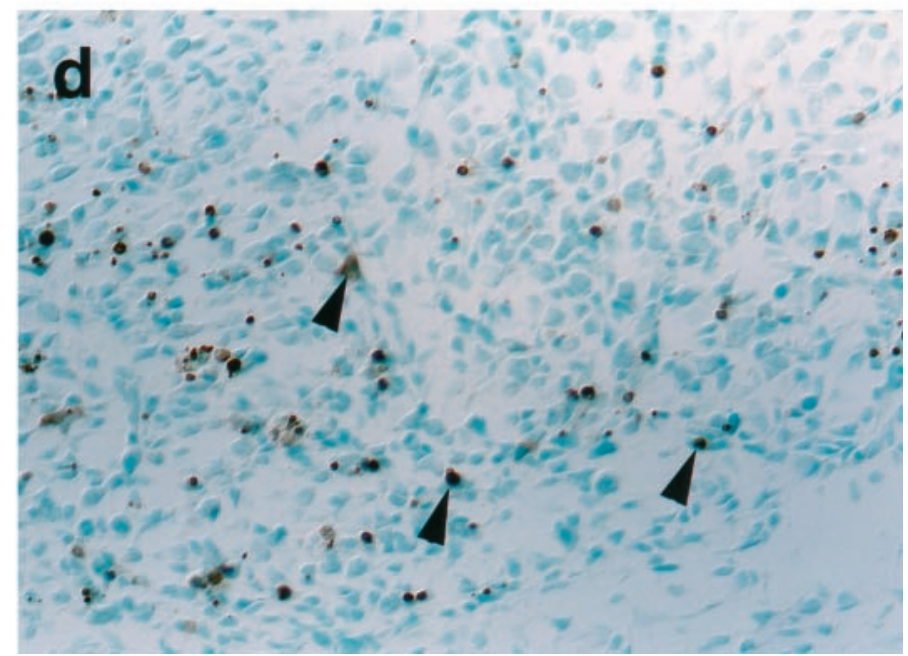

\section{c-jun-/-}

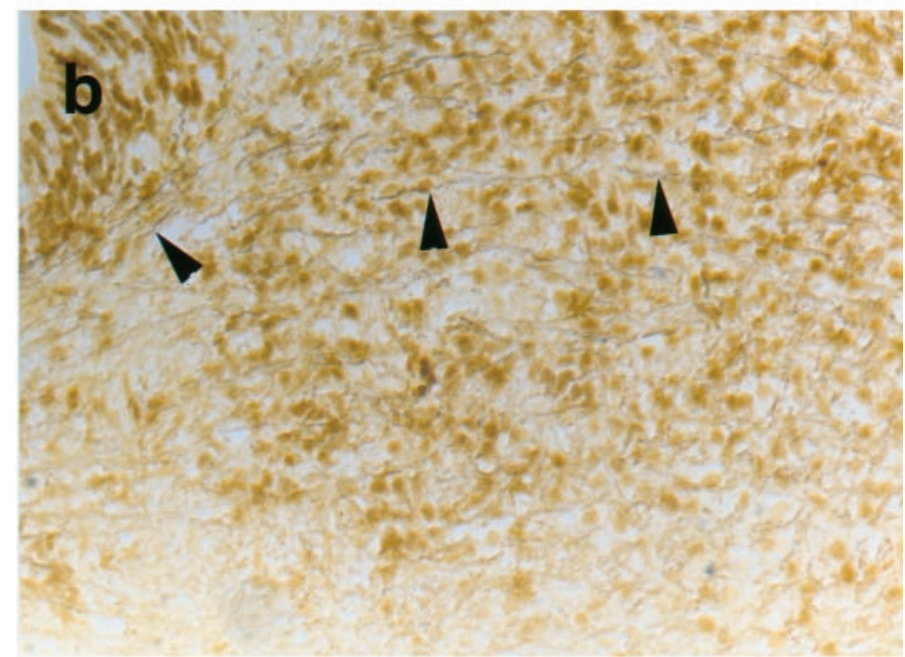

\section{Axogenesis in retinal grafts does not require a functional c-jun allele}

A preliminary characterization of the retinal grafts was performed using a thymosin- $\beta 10$-lac Z transgenic mouse (Chen and Morgan, unpublished data). In this mouse, the majority of retinal ganglion cells express $\beta$-galactosidase from the thymosin- $\beta 10$ promoter, which serves to define the optic nerve in vivo (Chen and Morgan, unpublished data). The use of this strain of transgenic mouse permits the unambiguous discrimination of donor axons from host axons. Eyes grafted from E12.5 thymosin- $\beta 10$-lacZ mouse embryos onto P0 rat brain continued to develop (Fig. 2a). By $13 \mathrm{~d}$ after transplantation the grafts had many characteristics of more mature eyes, including a lens and ganglion cell axonal bundles (Fig. $2 a$ ) that exited the graft and entered the retinorecipient layer of the superior colliculus (Fig. 2b). Because expression of c-jun has been specifically linked to regeneration of 

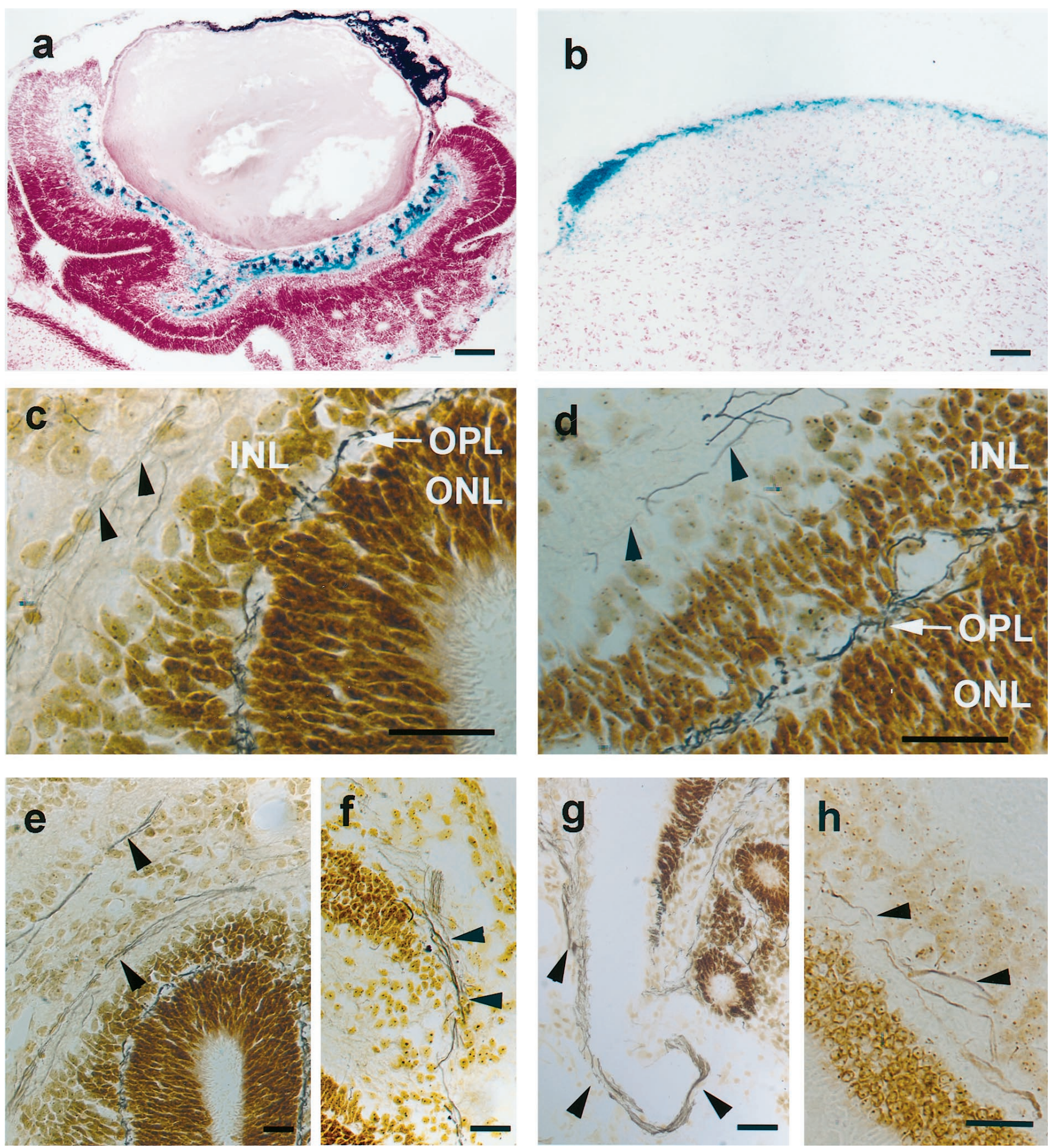

Figure 2. Axogenesis in retinal grafts from wild-type and c-jun-null embryos. $a$, A grafted eye from a thymosin- $\beta 10$-lacZ transgenic mouse develops retina-like structures and contains a lens. Note that thymosin- $\beta 10$-lacZ is present in retinal ganglion cells and their axons. $b$, Retinal ganglion cell axons of the thymosin- $\beta 10-$ lac Z graft project through the retinorecipient layer of the superior colliculus of the host. $c-h$, Grafts from wild-type (c, $e$, $g$ ) or c-jun knock-out $(d, f, h)$ mice were stained by Ungewitter's silver technique to reveal axons (arrows, arrowheads). Axons were evident in grafts from both ${\mathrm{c}-j u n^{+/+}}(c, e, g)$ and c-jun ${ }^{-1-}(d, f, h)$ mice. $c, d$, Axons growing through the inner plexiform and ganglion cell layers (black arrowheads) and the OPL (white arrow) in wild-type and c-jun-null grafts, respectively. In grafts of both genotypes, the INL and ONL are evident. In $e$, axons in the ganglion cell layer are evident (black arrowheads). In $f$, axons fasciculate into bundles (arrowheads), and in $g$ they can be seen leaving the graft and entering the superior colliculus of the host. $h$, c-jun-null graft 2 months after transplantation. Note that the general cytoarchitecture of the graft is maintained, and axons are still present in the outer plexiform layer (arrowheads). Scale bars: $a, b, g, 100 \mu \mathrm{m} ; c, d, 30 \mu \mathrm{m} ; e, f, h, 50 \mu \mathrm{m}$. 
ganglion cell axons (e.g., Herdegen et al., 1993; Hüll and Bähr, 1994a,b), retinal grafts from wild-type and c-jun-null embryos were next analyzed for axonal growth.

As shown in Figure 2, retinal grafts from both genotypes (equivalent to an in vivo retina at $\mathrm{P} 7$ ) had similar morphological appearances. Typically, the inner nuclear layer (INL) and outer nuclear layer (ONL) were evident as darkly stained regions separated by a thin acellular zone that represented the outer plexiform layer (OPL; Fig. 2c-e). Using Ungewitter's silver staining for fibers, axons were seen running through the outer plexiform layer (Fig. 2c,d, white arrows). The ganglion cell and optic fiber layers were less heavily stained but contained many axons that emanated from ganglion cells (Fig, 2e,g, black arrowheads). These fibers were frequently seen to form bundles (Fig. $2 f$ ) that exited the graft (Fig. $2 g$ ). There were no obvious differences in the incidence or organization of fibers in the outer plexiform or ganglion cell layers in grafts from the two genotypes (Fig. 2c, $d$ ). Therefore, c-jun was not essential for the gross formation of the retina or the initiation, elongation, and general path finding of axons in the retina.

Although c-Jun was not essential for axogenesis, it was conceivable that it might be necessary for the long-term maintenance of axonal integrity. Therefore, retinal transplants of both genotypes were allowed to develop for longer periods to determine whether axons degenerated selectively in c-jun-null grafts. Although older grafts of both genotypes generally started to show signs of infiltration, many still retained their anatomical integrity. Figure $2 h$ depicts a retinal graft from a c-jun-null embryo 2 months after transplantation. The graft had a well organized ONL, and the INL was still apparent. Furthermore, there were many axonal fibers in the outer plexiform layer (Fig. $2 h$, arrowheads). Equivalent grafts from wild-type embryos were indistinguishable from c-jun-null transplants (data not shown). Therefore, there was no evidence of selective long-term degeneration of axons in c-jun-null grafts.

\section{Relationship between c-jun-lacZ expression and apoptosis in developing and grafted retinas}

Although cell death in the developing retina is well documented (Young, 1984), it is not known whether this is recapitulated in retinal grafts. As demonstrated by others (Berko-Flint et al., 1994; Ren et al., 1997) c-Jun is expressed in the developing retina before, during, and after PCD (Fig. 3h). The authenticity of c-Jun is confirmed by the molecular mass $(\sim 39 \mathrm{kDa})$ of the immunoreactive band and the absence of the protein in extracts from c-jun-null embryos (Fig. 3h). Based on migration relative to standards for the dephospho and phospho forms of c-Jun, the predominant protein in the retina during development is dephospho-Jun. However, it remains to be established whether the gene is expressed in retinal transplants. To address these issues, cell death was monitored in both the developing retina and retinal grafts by conventional histology and the ISEL technique. Two independent methods were used to assess death, because ISEL may not detect all dying cells (Wood et al., 1993; Herrup and Busser, 1995). Although authentic c-Jun was detected in the retina by immunoblotting, the levels were not high enough to be convincingly demonstrated by immunohistochemistry. Therefore, the present cell death studies were performed using c-jun-lac Z transgenic mice (Chen and Morgan, unpublished data). In this strain of mice, $\beta$-galactosidase can be used as a very sensitive, surrogate indicator of c-jun expression in vivo. Details of the characteristics of these mice are to be presented elsewhere. In addition to the higher sensitivity, the strategy of monitoring Jun-lacZ rather than endogenous c-Jun was taken to preclude the possibility that c-jun was being expressed by occasional host cells that had infiltrated the graft. In addition, ISEL and $\beta$-galactosidase histochemistry are compatible techniques, permitting colocalization studies.

Because the vast majority of cell death in the mouse retina occurs postnatally, peaking between P7 and P9 (Young, 1984), the neonatal period was primarily examined. Pyknotic nuclei were detected in the retina of $\mathrm{P} 7 \mathrm{c}-\mathrm{jun}$-lac Z mice (Fig. 3a, inset). The dying cells were located primarily within the INL. Occasional pyknotic cells were observed at the boundary of the ONL where it juxtaposed the outer plexiform layer. Rarely were dead or dying cells seen in the ganglion cell layer (GCL) at this stage of development. A very similar anatomical pattern of cell death was observed using ISEL (Fig. $3 b$ ), confirming the data obtained by conventional histology (Fig. $3 a$ ). Consistent with previous studies (Young, 1984), there were few or no pyknotic cells in sections from P28 retinas (Fig. 3c).

Many Jun-lacZ-positive cells were seen throughout the ONL, INL, and GCL of the P7 retina (Fig. 3a,b). The distribution of labeling in the ONL was uniform, whereas only sporadic cells in the GCL expressed Jun-lacZ (Fig. 3a,b). Although there was substantial expression of Jun-lacZ within the INL, there appeared to be more labeling within the innermost region (Fig. $3 a$ ). When compared with ISEL-positive or pyknotic cells, many more cells expressed Jun-lacZ in the INL (Fig. 3a,b). Moreover, most pyknotic and apoptotic cells were negative for Jun-lacZ (Fig. 3a, inset). Quantitative analysis of apoptosis in the $\mathrm{P} 7$ retina revealed that there were $96.8 \pm 9.03$ pyknotic nuclei $/ \mathrm{mm}^{2}$. On average, only 2 pyknotic cells $/ \mathrm{mm}^{2}$ also expressed Jun-lacZ. It should be emphasized that this is merely a snapshot of cell death and Jun-lacZ expression. Because c-jun expression often increases and decreases rapidly, we cannot exclude the possibility that Jun-lacZ was expressed transiently before the cell becoming pyknotic. Furthermore, it is possible that the demise of these cells is accompanied by the proteolysis of Jun-lacZ. This could result in a loss of $\beta$-galactosidase staining, although c-Jun might still be expressed.

In the P28 retina, Jun-lacZ-positive cells were still evident in the INL and GCL (Fig. 3c) despite the fact that there is no PCD at this time (Young, 1984; Fig. 3c). As at P7, only sporadic cells were labeled in the GCL at P28. However, two bands of cells, located at the inner and outer boundaries of the INL, expressed Jun-lacZ at this stage (Fig. $3 c$ ).

In view of the discrepancies between Jun-LacZ expression and PCD, additional times of development were investigated. At E11.5, there was weak expression of Jun-lacZ in the neuroblast layer (Fig. 3e). In addition, there was prominent expression of Jun-lacZ in what appeared to be migrating ganglion cells (Sidman, 1961) (Fig. 3e, inset). There were very few pyknotic cells evident in the early retina (Fig. 3e). At E17.5, there was still weak expression of Jun-lacZ in the neuroblast population, but the band of presumptive migrating cells was no longer evident (Fig. 3f). However, Jun-lacZ-positive cells were seen in the ganglion cell layer at this time (Fig. $3 f$ ). In addition, there were Jun-lacZexpressing cells associated with the proximal portion of the optic nerve (Fig. 3f). Only occasional pyknotic cells were seen at E17.5 (Fig, $3 f$ ).

These data indicated that (1) like c-Jun, Jun-lacZ was expressed in the developing retina: (2) Jun-lacZ expression was more widespread than cell death in the retina; (3) Jun-lacZ was 

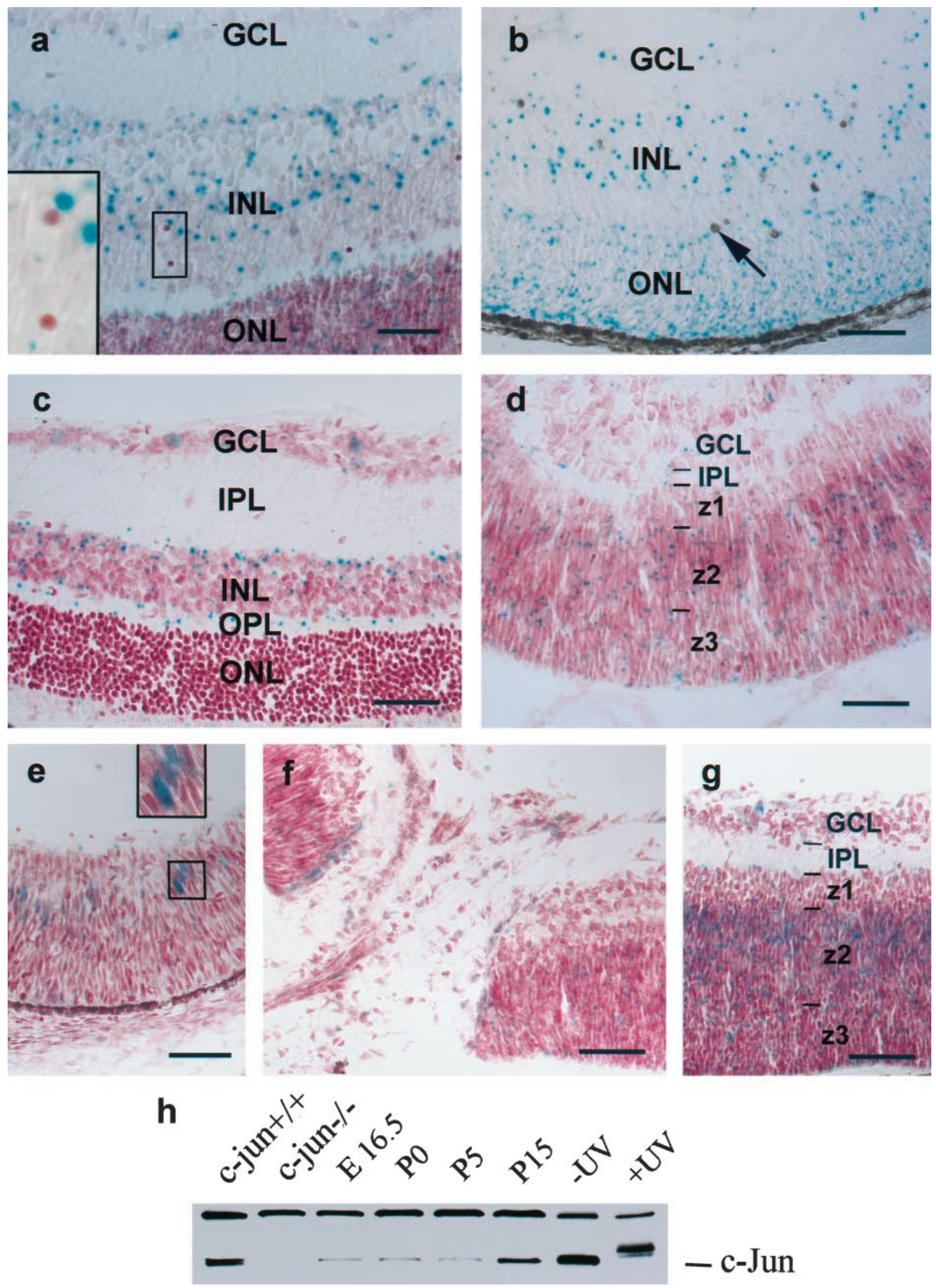

Figure 3. c-jun-lac $\mathrm{Z}$ is expressed in normal and in grafted retinas before, during, and after the period of developmental cell death. $a$, A retina from a P7 c-jun-lacZ transgenic mouse was processed for $\beta$-galactosidase histochemistry and counterstained with neutral red to detect pyknotic nuclei. The X-gal product (blue) is visible in all cell layers of the retina, whereas pyknotic nuclei (dark red, inset) are predominantly located in the INL. $b$, A P7 retina from a c-jun-lac Z mouse was processed for $\beta$-galactosidase histochemistry followed by the ISEL procedure. Jun-lacZ is expressed throughout the retina, whereas ISEL-positive cells (brown reaction product) are only observed within the INL and adjacent to the OPL (arrow). (Figure legend continues) 
only rarely detected in retinal cells with pyknotic nuclei, although it may have been expressed at some point before their formal demise; and (4) Jun-lacZ expression was observed in retinal cells at P28 that were not destined to die.

Having established the relationship between Jun-lacZ expression and PCD in the retina in vivo, a parallel analysis was performed in retinal grafts. Figure $3 d$ shows a retinal xenograft from a c-jun-lac Z mouse $12 \mathrm{~d}$ after transplantation. This time point was calculated as being equivalent to an in vivo retina at $\mathrm{P} 3$, which is shown in Figure $3 g$ for comparison. There was widespread expression of Jun-lacZ in both the normal retina and the graft (Fig. $3 d, g$ ). The various layers of the retinal graft were not as well defined as at later stages (compare Figs. $3 d, 2 c, g$ ). The darkest stained region of the transplant was strongly Jun-lacZ-positive (Fig. 3d) and was composed of three subzones (Fig. 3d, z1-z3) that were also evident in the $\mathrm{P} 3$ retina in vivo (Fig. $3 g$ ). The innermost region (z1) is known to comprise predominantly differentiating and dying amacrine cells that will contribute to the INL (Young, 1984, 1985). Although this zone contained only a few Jun-lacZpositive cells, the majority of the pyknotic cells present in the graft were seen in $\mathrm{z} 1$ (Figs. $3 d, 4 a, b$ ). The most intense staining for Jun-lacZ was evident in z2 and z3. The z2 and z3 regions contain neuroblasts and differentiating neurons that give rise to the INL and ONL (Young, 1985). Consistent with previous studies (Young 1984; see Fig. 3e,g), there were few pyknotic cells in the neuroblast population (Figs. $3 d-f, 4 a, b$ ). The z3 region contains predominantly photoreceptors and neuroblasts at P3 (Caley et al., 1972). As in the $\mathrm{z} 2$ region, there were few pyknotic cells in the future ONL despite the fact that jun-lac Z was widely expressed (Figs. 3d, 4a,b). Both the graft and the P3 retina had detectable inner plexiform layers (Fig. $3 d, g$ ). Beyond the IPL, a somewhat diffuse GCL was seen in the transplant (Fig. $3 d$ ). Sporadic Jun-lacZ-positive and pyknotic cells were observed in the GCL of the P3 retina and the presumptive GCL of the graft (Figs. 3d,g, 4a,b). However, the two were rarely coincident, and there were more Jun-lacZ-positive cells than apoptotic cells (Figs. $3 d, 4 a, b)$.

These studies established that retinal grafts did express JunlacZ but that this was not specifically associated with regions of the retina undergoing PCD.

\section{c-jun is not essential for PCD in retinal grafts}

PCD in the mouse retina is largely confined to the perinatal and early postnatal period (Young, 1984). Therefore, grafts from wild-type and c-jun-null littermates that were equivalent to P3, P7, and P9 were examined for spontaneous cell death.

At presumptive P3, the majority of cells with pyknotic nuclei were located in the region of differentiating INL cells and in the GCL (Figs. 3d, 4a,b, insets). In P3 retinas in vivo, PCD occurs predominantly among the ganglion and amacrine cell populations
(Young, 1984). Thus, the location of the cells with pyknotic nuclei in the retinal transplants was consistent with ganglion and amacrine cells. Moreover, there were no overt differences in the numbers of pyknotic nuclei in grafts from wild-type $(57.3 \pm 8.8$ pyknotic nuclei $/ \mathrm{mm}^{2}$ ) or c-jun-null embryos $(58.3 \pm 7.5$ pyknotic nuclei $/ \mathrm{mm}^{2}$ ) (Fig. 4a,b). These numbers from presumptive P3 grafts are in good agreement with the number of pyknotic nuclei observed in the $\mathrm{P} 3$ retina in vivo $(47.7 \pm 11.6$ pyknotic nuclei/ $\mathrm{mm}^{2}$ ) (Table 1).

In grafts at presumptive $\mathrm{P} 7$, the neuroblasts had largely differentiated to give a well defined ONL and a more diff use INL (Fig. $4 c, d)$. At this stage, only occasional apoptotic cells were seen at the inner boundary of the ONL (Fig. 4c,d). This is consistent with ISEL staining in the $\mathrm{P} 7$ retina in vivo, where only sporadic ISEL-positive and pyknotic cells were seen at the border of the ONL where it juxtaposed the outer plexiform layer (compare Figs. $3 b, 4 c, d)$. Young (1984) reported dying rods located near the inner boundary of the ONL. Thus, the occasional apoptotic figures may represent degenerating rods. More ISEL-positive cells were evident within the INL of presumptive P7 grafts, where they tended to lie close to the boundary with the inner plexiform layer (Fig. 4c,d). Again, the frequency and distribution of the ISEL-positive cells was generally the same as that observed in vivo at this age (compare Figs. $3 b$ Fig. $4 c, d$ ). This is consistent with the reported peak of death within the INL of bipolar cells, inner rod cells, and Müller glia (Young, 1984). As in presumptive P3 grafts, there were no obvious differences in the position or frequency of ISEL-positive cells between the two genotypes (Fig. $4 c, d)$. Wild-type grafts contained $46.8 \pm 9.8$ pyknotic nuclei/ $\mathrm{mm}^{2}$, whereas retinal grafts from c-jun-null embryos contained $41.6 \pm 8.0$ pyknotic nuclei $/ \mathrm{mm}^{2}$.

At presumptive P9, the grafts had matured further, and the previously diffuse INL had become more defined (Fig. 4e,f). Apoptosis in grafts at presumptive P9 was essentially the same as at presumptive P7, with ISEL-positive cells being observed predominantly in the INL (Fig. 4e,f). In vivo at P9, most cell death occurs within the INL, where Müller glia, bipolar cells, and, to a lesser extent, inner rods die (Young, 1984). As at earlier stages of maturation, there were no marked differences in the frequency or location of apoptotic cells in grafts from wild-type or c-jun-null embryos (Fig. 4e,f). Wild-type grafts contained $87.7 \pm 27.8$ pyknotic nuclei $/ \mathrm{mm}^{2}$, whereas retinal grafts from c-jun-null embryos contained $82.4 \pm 15.5$ pyknotic nuclei $/ \mathrm{mm}^{2}$.

The foregoing data indicated that c-jun was not essential for neuronal death in vivo up to E12.5 or for PCD in retinal grafts up to the equivalent of P9. Moreover, both the in vivo and transplantation data indicated that axon growth occurred independently of c-Jun function.

\section{$\longleftarrow$}

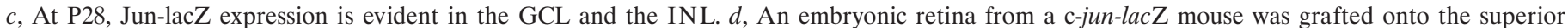

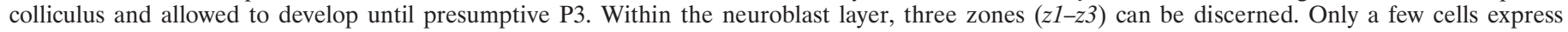

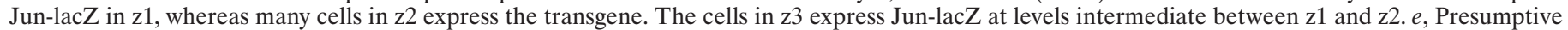

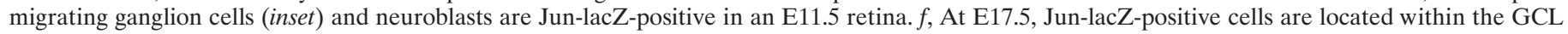

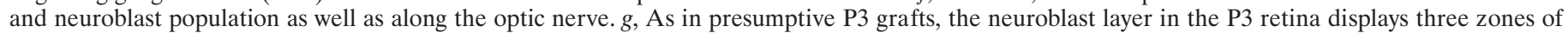

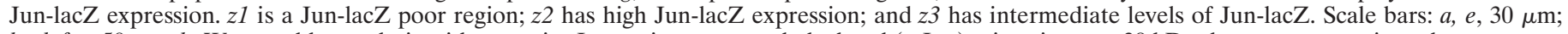

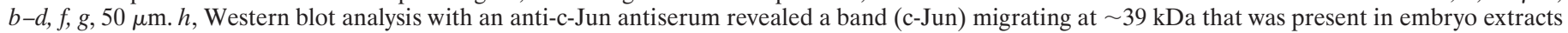

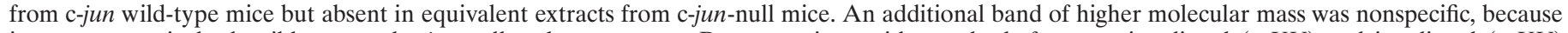

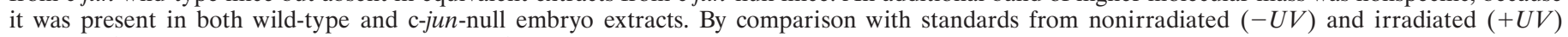

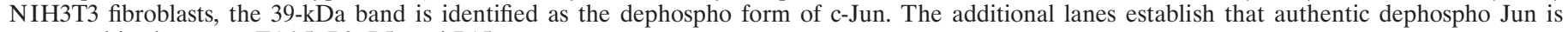
expressed in the eye at E16.5, P0, P5, and P15. 

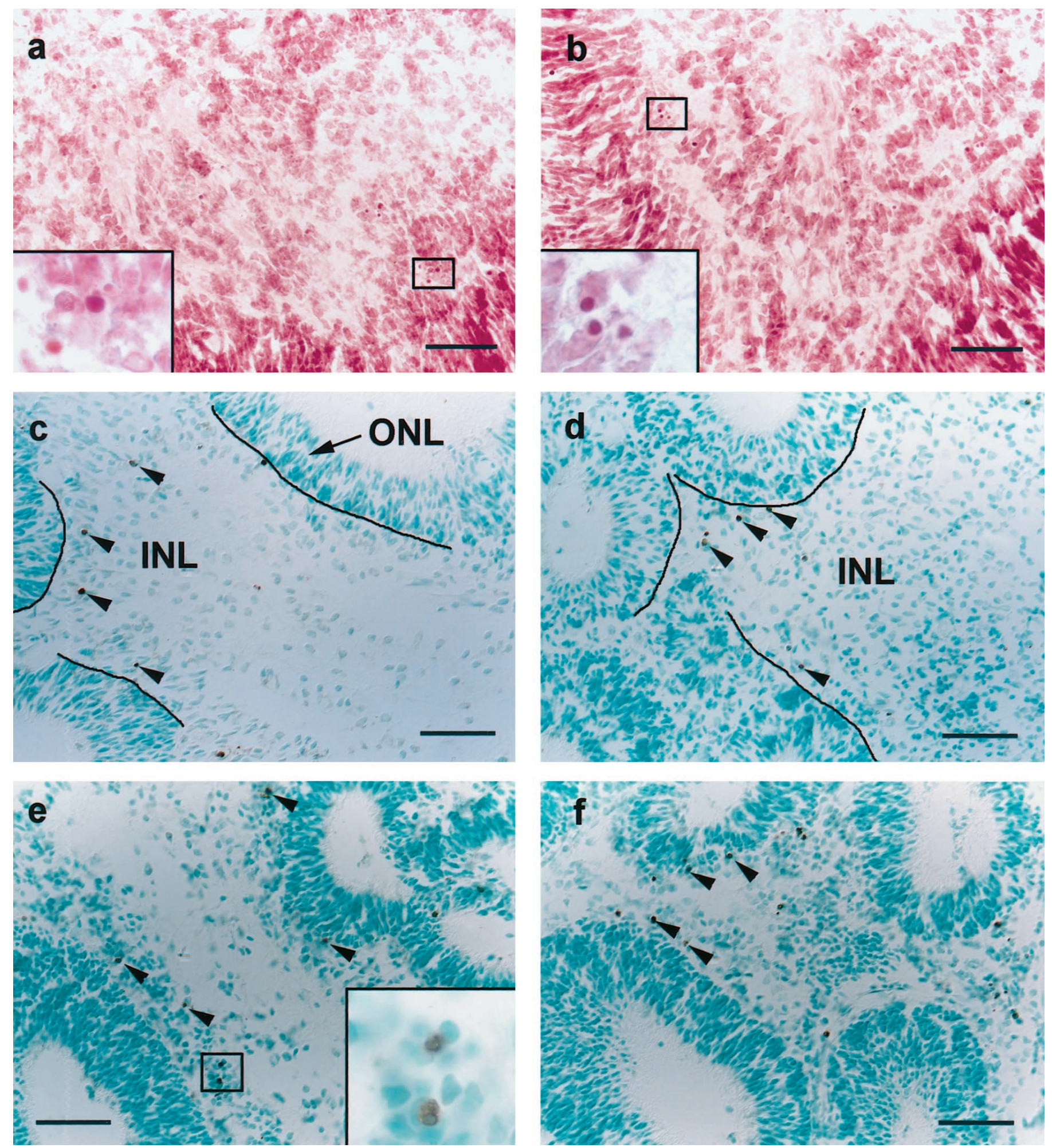

Figure 4. Cell death in retinal transplants of wild-type and c-jun knock-out mice. Retinas from c-jun ${ }^{+/+}(a)$ and c-jun $^{-1-}(b)$ E13.5 mice were grafted onto the superior colliculus and allowed to develop until a stage corresponding to P3. In neutral red-stained sections, darker areas correspond to the neuroblast layer, whereas lighter areas represent the GCL and the inner part of the INL. Pyknotic nuclei (dark red cell fragments, inset) are predominantly detected in the lightly stained areas. $c, d$, Retinas from c-jun ${ }^{+/}(c)$ and c-jun ${ }^{-1-}(d)$ E12.5 mice were transplanted to day-of-birth rats and allowed to develop until the equivalent of P7, when cell death was detected by ISEL. In these methyl green-counterstained sections, the ONL appears as a dark green area, whereas the INL is light green. In both c-jun ${ }^{+/}(c)$ and c-jun ${ }^{-1-}(d)$ transplants, ISEL-positive cells (arrowheads) are situated within the INL and occasionally immediately above the ONL. A line has been added to show the demarcation between the ONL and INL. $e, f$, Additional grafts from c-jun ${ }^{+/}$ $(e)$ and c-jun $^{-1-}(f)$ E13.5 embryos were allowed to develop to the equivalent of P9. The INL is now more distinct and contains the majority of the ISEL-positive cells (arrowheads). e, inset, Enlargement of the boxed area showing ISEL-positive cells in the INL. There are no obvious differences between wild-type $(e)$ and c-jun-null $(f)$ grafts with regard to the location or approximate numbers of ISEL-positive cells. Scale bars: $a, b, 30 \mu \mathrm{m} ; c-f, 50 \mu \mathrm{m}$. 


\begin{tabular}{|c|c|c|}
\hline \multirow{2}{*}{$\begin{array}{l}\text { Presumptive postnatal } \\
\text { (d) }\end{array}$} & \multicolumn{2}{|c|}{ Pycnotic nuclei $/ \mathrm{mm}^{2}$} \\
\hline & c-jun ${ }^{+/+}$grafts & c-jun ${ }^{-l-}$ grafts \\
\hline P3 & $57.3 \pm 8.8$ & $58.3 \pm 7.5$ \\
\hline P7 & $46.8 \pm 9.8$ & $41.6 \pm 8.0$ \\
\hline P9 & $87.7 \pm 27.8$ & $82.4 \pm 15.5$ \\
\hline $\mathrm{P} 3$ retina in vivo & $47.7 \pm 11.6$ & NA \\
\hline
\end{tabular}

Pycnotic nuclei were counted in serial sections through the center of the grafts or the midline of the retina in vivo. Numbers represent average pycnotic nuclei per square millimeter of at least five sections \pm SEM (see Materials and Methods). For each genotype, four independent grafts were analyzed. NA, Not applicable.

\section{DISCUSSION}

Previous studies have demonstrated an association between the expression of c-jun and the apparently opposing phenomena of programmed neuronal death (Dragunow et al., 1993; Anderson et al., 1995; Ferrer et al., 1996; Guégan et al., 1997) and neuronal regeneration (Jenkins and Hunt, 1991; Leah et al., 1991; Jenkins et al., 1993; Vaudano et al., 1998). Although the evidence for c-Jun playing an active role in regeneration is largely circumstantial, several reports have directly implicated this basic zipper transcription factor in PCD in culture (Estus et al., 1994; Ham et al., 1995). However, a study in midgestational c-jun-null embryos questioned the role of this gene in programmed cell elimination in vivo (Roffler-Tarlov et al., 1996). This discrepancy could result from there being mechanistically distinct forms of neuronal death. Indeed, it has been proposed that apoptosis among dividing neuroblasts in neurogenic regions of the nervous system is distinct from that occurring after the neuroblasts exit the cell cycle and become subject to target-dependent selection or synaptic competition (Blaschke et al., 1996). Although some targetdependent PCD may be occurring in both the trigeminal and dorsal root ganglia at E12.5 (Davies and Lumsden, 1984; White et al., 1996), this process is most prominent after the time that c-jun-null embryos die (e.g., Wright et al., 1983; Young, 1984; Herrup and Busser, 1995). Therefore, it was unclear whether c-jun might play a critical role in PCD during later development. In an attempt to reconcile the culture data with the in vivo results, a retinal grafting paradigm was used to extend the analysis of PCD to encompass times when the second type of cellular demise occurred. Moreover, by characterizing PCD, gene expression, and axogenesis in retinal grafts from transgenic and c-jun nullizygous mice, it was possible to assess the impact of loss of c-Jun function on multiple aspects of retinal phenotype.

The retinal transplantation paradigm used here has been extensively characterized at the anatomical and physiological levels (McLoon and Lund, 1980; Hankin and Lund, 1987). However, it was unknown whether these grafts exhibited temporally and spatially appropriate PCD or spontaneously expressed c-jun. As shown here, retinas in vivo and retinal grafts at the equivalent stages of maturation have indistinguishable patterns of PCD and c-jun-lacZ expression. Furthermore, the location of the dying cells is consistent with the known pattern of PCD in the retina (Young, 1984). The grafts also have the same general cytoarchitecture as normal retinas and produce axons, axonal bundles, and presumptive optic nerves; i.e., the transplants behaved largely like retinas in vivo with regard to anatomical development, axogenesis, axonal path finding, and PCD. Therefore, these grafts do represent an appropriate model for investigating the role of c-jun in PCD and axonal growth.
Although the expression patterns of c-jun-lacZ in retinas and retinal grafts were similar, they were not coincident with PCD. For example, Jun-lacZ was rarely detected in pyknotic or ISELpositive cells. In addition, many cells expressed c-jun-lac Z that were not destined to die. Besides a lack of correlation between expression of c-jun-lac Z and cell death, the level and location of PCD in retinal grafts from c-jun-null embryos were indistinguishable from those of wild-type transplants. Thus, despite several compelling studies indicating that c-Jun plays a causal role in growth factor-dependent death in culture (Colotta et al., 1992; Estus et al., 1994; Ham et al., 1995), the present transplantation study establishes that c-jun is not essential for neuronal PCD in the retina. In addition, c-jun is not essential for PCD in the peripheral (e.g., trigeminal ganglion) or central nervous systems (e.g., telencephalon) in vivo up to E12.5.

A number of features may account for the discrepancies in c-jun function between the in vivo and in vitro studies. First, the studies implicating c-Jun causally in neuronal PCD all involved cultures of sympathetic neurons. Because the present investigation primarily studied the retina, we cannot discount the possibility that c-Jun plays a unique role in eliminating sympathetic neurons. In this case, there may be more heterogeneity in the suicide pathway than previously thought. Second, it is possible that growth factor-dependent neuronal death in culture may have mechanistic differences from PCD in vivo. Conversely, grafting may alter the underlying mechanisms of PCD in the retina, making them independent of c-Jun. Fourth, the discrepancy may also be a result of the temporal limitations of investigating PCD in culture. Thus, antagonizing c-Jun function in vitro may give the impression of halting PCD, whereas in reality it may only have retarded the process. Fifth, the absence of a functional c-jun allele in vivo may result in the upregulation of a related gene with a redundant function. It should also be emphasized that c-jun could still play a nonessential role in PCD in vivo and in vitro.

Although the distribution of Jun-lacZ expression was not predictive of PCD, the transgene was expressed in retinal neurons that were undergoing axon growth and synaptogenesis both in vivo and in transplants. Although PCD is confined to a relatively narrow time window in the retina, axon growth and synaptogenesis span a longer developmental period, beginning in midgestation and ending after P20 (Olney, 1968a,b; Hinds and Hinds, 1974). In contrast, PCD in the retina begins around the time of birth, peaks at approximately P8, and is largely finished by P12 (Young, 1984). The spatial and temporal expression of Jun-lacZ mirrored axogenesis and synaptogenesis. For example, ganglion cells elaborate their axons from E12.5 onward (Hinds and Hinds, 1974), and Jun-lacZ was expressed in the region of the retina that contained differentiating ganglion cells during this period. By P7 there is extensive axogenesis and synaptogenesis of bipolar cells in the INL (Olney, 1968a,b) that contribute to the formation of the OPL. At P7 there was also substantial expression of Jun-lacZ within the INL. This finding is consistent with reports linking expression of c-jun to axonal sprouting in many brain regions, including the retina (Herdegen et al., 1993; Hüll and Bähr, 1994b; Robinson, 1994, 1995, 1996; Vaudano et al., 1998). However, Jun-lacZ was also expressed in neuroblasts and photoreceptors, which do not possess axons. Therefore, the association between Jun-lacZ expression and axogenesis in the developing retina was not absolute. This finding extends one study of Jun expression in the adult retina after optic nerve transection in which Jun-like immunoreactivity was not invariably associated with sprouting ganglion cells (Isenmann and Bähr, 1997). 
Unlike PCD, there are no experiments that have directly addressed whether c-Jun function is necessary for axon growth. It was impossible to investigate neuronal regeneration using c-junnull xenografts. However, axonal regeneration in the adult shares a number of common molecular features with axogenesis during development, and as shown here, c-jun-lac $\mathrm{Z}$ is expressed during axogenesis. Therefore, the role of c-Jun in developmental axon growth was investigated in grafts from wild-type and c-jun-null mice. The absence of a functional c-jun allele had no discernible effect on the initiation, elongation, or path finding of axons in vivo or in transplants. In addition, there were no obvious differences in the number or appearance of axons or axon bundles in the brains or retinal xenografts of either genotype. Finally, the gross morphological characteristics of axons and axon bundles in 2-monthold c-jun-null retinal grafts were indistinguishable from wild-type grafts, suggesting that c-Jun was not essential for the maintenance of axonal integrity.

As for PCD, a number of limitations in the study have to be acknowledged. First, although developmental axogenesis shares many molecular and structural features with axonal regeneration in the adult, the processes are not identical. For example, adult axons are myelinated, and the extracellular milieu of the adult CNS is nonpermissive for axon growth (Ramon y Cajal, 1959; Schnell and Schwab, 1990). Therefore, the present data cannot exclude the possibility that c-jun is essential for adult nerve regeneration. Second, c-jun may still play a dispensable role in developmental axogenesis. Third, because this study involved a light microscopic analysis of axogenesis, there may be as yet unidentified functional, biochemical, or ultrastructural deficits in neurons in c-jun-null mice.

The present study also indicated that Jun-lacZ was expressed in retinal neuroblasts during development. This finding is consistent with a previous observation of c-jun expression in many of the proliferating populations in the developing nervous system (Wilkinson et al., 1989). However, as with PCD and axon growth, this association was not complete, and there were no obvious deficiencies in the neuroblast populations in jun-null embryos or grafts. Therefore, the expression of c-jun and c-jun-lac Z was not absolutely correlated with, or overtly necessary for, any particular cell fate or developmental event in the retina. Rather, c-jun seems to fulfill a pleiotypic role, perhaps contributing in a nonessential way to multiple biological processes. Nevertheless, this gene may still serve as a valuable reporter with which to identify the signaling molecules and pathways that are activated during death and regeneration in the nervous system.

\section{REFERENCES}

Anderson AJ, Pike CJ, Cotman CW (1995) Differential induction of immediate early gene proteins in cultured neurons by $\beta$-amyloid $(\mathrm{A} \beta)$ : association of c-Jun with $\mathrm{A} \beta$-induced apoptosis. J Neurochem 65:1487-1498.

Berko-Flint Y, Levkowitz G, Vardimon L (1994) Involvement of c-Jun in the control of glucocorticoid receptor transcriptional activity during development of chicken retinal tissue. EMBO J 13:646-654.

Blaschke AJ, Staley K, Chun J (1996) Widespread programmed cell death in proliferative and postmitotic regions of the fetal cerebral cortex. Development 122:1165-1174.

Bossy-Wetzel E, Bakiri L, Yaniv M (1997) Induction of apoptosis by the transcription factor c-Jun. EMBO J 16:1695-1709.

Caley DW, Johnson C, Liebelt RA (1972) The postnatal development of the retina in the normal and rodless CBA mouse: a light and electron microscopic study. Am J Anat 133:179-212.

Colotta F, Polentarutti N, Sironi M, Mantovani, A (1992) Expression and involvement of c-fos and c-jun protooncogenes in programmed cell death induced by growth factor deprivation in lymphoid cell lines. J Biol Chem 267:18278-18283.

Davies A, Lumsden A (1984) Relation of target encounter and neuronal death to nerve growth factor responsiveness in the developing mouse trigeminal ganglion. J Comp Neurol 223:124-137.

DeFelipe C, Hunt SP (1994) The differential control of c-jun expression in regenerating sensory neurons and their associated glial cells. J Neurosci 14:2911-2923.

Dragunow M, Young D, Hughes P, MacGibbon G, Lawlor P, Singleton K, Sirimanne E, Beilharz E, Gluckman P (1993) Is c-Jun involved in nerve cell death following status epilepticus and hypoxic-ischaemic brain injury? Mol Brain Res 18:347-352.

Estus S, Zaks WJ, Freeman RS, Gruda M, Bravo R, Johnson Jr EM (1994) Altered gene expression in neurons during programmed cell death: Identification of c-jun as necessary for neuronal apoptosis. J Cell Biol 127:1717-1727.

Ferrer I, Olivé M, Blanco R, Cinós C, Planas AM (1996) Selective c-Jun overexpression is associated with ionizing radiation-induced apoptosis in the developing cerebellum of the rat. Mol Brain Res 38:91-100.

Gavrieli Y, Sherman Y, Ben-Sasson SA (1992) Identification of programmed cell death in situ via specific labeling of nuclear DNA fragmentation. J Cell Biol 119:493-501.

Gorgels TG, Oestreicher AB, de Kort EJ, Gispen WH (1987) Immunocytochemical distribution of the protein kinase C substrate B-50 (GAP43) in developing rat pyramidal tracts. Neurosci Lett 16:59-64.

Guégan C, Lévy V, David J-P, Ajchenbaum-Cymbalista F, Sola B (1997) c-Jun and cyclin D1 proteins as mediators of neuronal death after a focal ischaemic insult. NeuroReport 8:1003-1007.

Ham J, Babij C, Whitfield J, Pfarr CM, Lallemand D, Yaniv M, Rubin LL (1995) A c-Jun dominant negative mutant protects sympathetic neurons against programmed cell death. Neuron 14:927-939.

Hankin MH, Lund RD (1987) Role of the target in directing the outgrowth of retinal axons: transplants reveal surface-related and surfaceindependent cues. J Comp Neurol 263:455-466.

Herdegen T, Bastmeyer M, Bähr M, Stuermer C, Bravo R, Zimmermann M (1993) Expression of JUN, KROX, and CREB transcription factors in goldfish and rat retinal ganglion cells following optic nerve lesion is related to axonal sprouting. J Neurobiol 24:528-543.

Herrup K, Busser JC (1995) The induction of multiple cell cycle events precedes target-related neuronal death. Development 121:2385-2395.

Hilberg F, Aguzzi A, Howells N, Wagner EF (1993) c-Jun is essential for normal mouse development and hepatogenesis. Nature 365:179-181.

Hinds JW, Hinds PL (1974) Early ganglion cell differentiation in the mouse retina: an electron microscopic analysis utilizing serial sections. Dev Biol 37:381-416.

Horsburgh GM, Sefton AJ (1987) Cellular degeneration and synaptogenesis in the developing retina of the rat. J Comp Neurol 263:553-566.

Hüll M, Bähr M (1994a) Differential regulation of c-JUN expression in rat retinal ganglion cells after proximal and distal optic nerve transection. Neurosci Lett 178:39-42.

Hüll M, Bähr M (1994b) Regulation of immediate-early gene expression in rat retinal ganglion cells after axotomy and during regeneration through a peripheral nerve graft. J Neurobiol 25:92-105.

Isenmann S, Bähr M (1997) Expression of c-Jun protein in degenerating retinal ganglion cells after optic nerve lesion in rat. Exp Neurol 147:28-36.

Jenkins R, Hunt SP (1991) Long-term increase in the levels of c-jun mRNA and JUN protein-like immunoreactivity in motor and sensory neurons following axon damage. Neurosci Lett 129:107-110.

Jenkins R, Tetzlaff W, Hunt SP (1993) Differential expression of immediate early genes in rubrospinal neurons following axotomy in the rat. Eur J Neurosci 5:203-209.

Johnson RS, van Lingen B, Papaioannou VE, Spiegelman BM (1993) A null mutation at the c-jun locus causes embryonic lethality and retarded cell growth in culture. Genes Dev 7:1309-1317.

Koistinaho J, Hicks KJ, Sagar SM (1993) Long-term induction of c-jun mRNA and Jun protein in rabbit retinal ganglion cells following axotomy or colchicine treatment. J Neurosci Res 34:250-255.

Leah J, Herdegen T, Kovary K, Bravo R (1991) Selective expression of JUN proteins following peripheral axotomy and axonal transport block in the rat: evidence for a role in the regeneration process. Brain Res 566:198-207.

McLoon SC, Lund RD (1980) Specific projections of retina transplanted to rat brain. Exp Brain Res 40:273-282.

Olney JW (1968a) An electron microscopic study of synapse formation, 
receptor outer segment development, and other aspects of developing mouse retina. Invest Ophthalmol 7:250-268.

Olney JW (1968b) Centripetal sequence of appearance of receptorbipolar synaptic structures in developing mouse retina. Nature 218:281-282.

Oppenheim RW (1991) Cell death during development of the nervous system. Annu Rev Neurosci 14:453-501.

Pfenninger KH, de la Houssaye BA, Helmke SM, Quiroga S (1991) Growth regulated proteins and neuronal plasticity. A commentary. Mol Neurobiol 5:143-151.

Ramon y Cajal S (1959) Degeneration and regeneration of the nervous system. New York: Hafner.

Ren Y, Holdengreber V, Ben-Shaul Y, Shah BH, Varanasi J, Hausman RE (1997) Causal role for jun protein in the stimulation of choline acetyltransferase by insulin in embryonic chick retina. Biochem Biophys Res Commun 27:788-93.

Robinson GA (1994) Immediate early gene expression in axotomized and regenerating retinal ganglion cells of the adult rat. Mol Brain Res 24:53-54.

Robinson GA (1995) Axotomy-induced regulation of c-Jun expression in regenerating rat retinal ganglion cells. Mol Brain Res 30:61-69.

Robinson GA (1996) Changes in the expression of transcription factors ATF-2 and Fra-2 after axotomy and during regeneration in rat retinal ganglion cells. Mol Brain Res 41:57-64.

Roffler-Tarlov S, Brown JJG, Tarlov E, Stolarov J, Chapman DL, Alexiou M, Papaioannou VE (1996) Programmed cell death in the absence of c-Fos and c-Jun. Development 122:1-9.

Schnell L, Schwab M (1990) Axonal regeneration in the rat spinal cord produced by an antibody against myelin-associated neurite growth inhibitors. Nature 343:269-272.

Sidman RL (1961) Histogenesis of mouse retina studied with thymidine-
$\mathrm{H}^{3}$. In: The structure of the eye (Smelser GK, ed), pp 487-505. New York: Academic.

Ungewitter LH (1951) A urea silver nitrate method for nerve fibres and nerve endings. Stain Technol 26:73-76.

Van der Zee CE, Nielander HB, Vos JP, Lopes da Silva S, Verhaagen J, Oestreicher AB, Schrama LH, Schotman P, Gispen WH (1989) Expression of growth-associated protein B-50 (GAP-43) in dorsal root ganglia and sciatic nerve during regenerative sprouting. J Neurosci 9:3505-3512.

Vaudano E, Campbell G, Hunt SP, Lieberman AR (1998) Axonal injury and peripheral nerve grafting in the thalamus and cerebellum of the adult rat: upregulation of c-jun and correlation with regenerative potential. Eur J Neurosci 10:2644-2656.

White FA, Silos-Santiago I, Molliver DC, Nishimura M, Phillips H, Barbacid M, Snider WD (1996) Synchronous onset of NGF and TrkA survival dependence in developing dorsal root ganglia. J Neurosci 16:4662-4672.

Wilkinson DG, Bhatt S, Ryseck R-P, Bravo R (1989) Tissue-specific expression of c-jun and jun $\mathrm{B}$ during organogenesis in the mouse. Development 106:465-471.

Wood KA, Dipasquale B, Youle RJ (1993) In situ labeling of granule cells for apoptosis-associated DNA fragmentation reveals different mechanisms of cell loss in developing cerebellum. Neuron 11:621-632.

Wright LL, Cunningham, TJ, Smolen AJ (1983) Developmental neuron death in the rat superior cervical sympathetic ganglion: cell counts and ultrastructure. J Neurocytol 12:727-738.

Young RW (1984) Cell death during differentiation of the retina in the mouse. J Comp Neurol 229:362-373.

Young RW (1985) Cell differentiation in the retina of the mouse. Anat Rec 212:199-205. 